\title{
Hepatocellular Carcinoma Arising from Hepatocellular Adenoma in an Elderly Male Patient
}

\author{
Manuel Lim, Jong Man Kim, Ji Eun Kwon, Eun Sung Jeong, Jaehun Yang, Okjoo Lee, Kyeong Deok Kim, Sang Jin Kim, \\ Jinsoo Rhu, Gyu-Seong Choi, Jae-Won Joh \\ Department of Surgery, Samsung Medical Center, Sungkyunkwan University School of Medicine, Seoul, Korea
}

Received Dec. 29, 2020

Revised Mar. 2, 2021

Accepted Mar. 2, 2021
Hepatocellular adenoma is a benign tumor of the liver occurring predominantly in young women taking oral contraceptives. The malignant transformation of hepatocellular adenoma into hepatocellular carcinoma has rarely been reported. Herein, we report the case of an elderly male patient with hepatocellular carcinoma that developed from hepatocellular adenoma. The patient's high risk for surgery and conflicting biopsy and imaging results made it difficult to determine the treatment direction. Eventually, the mass was completely removed by laparoscopic left hemi-hepatectomy without complications. (J Liver Cancer 2021;21:87-91)

Keywords: Hepatocellular adenoma; Hepatocellular carcinoma; Aged; Minimal invasive surgery

\section{INTRODUCTION}

Hepatocellular adenoma (HCA) is a rare benign liver tumor that usually occurs in women who use oral contraceptives. ${ }^{1} \mathrm{HCA}$ is caused by the benign proliferation of hepatocytes with high glycogen and fat contents and is stimulated by hormonal or metabolic abnormalities. ${ }^{2,3}$ Clinical manifestations of this pathology include asymptomatic presentation, abdominal pain, abdominal mass, or hemorrhage (in case of rupture). HCA is usually detected incidentally in imaging studies in asymptomatic patients. Although the pathophysiology of the transformation of HCA to hepatocellular carcinoma (HCC) is not fully known, the rate of transformation

\section{Corresponding author : Jong Man Kim}

Department of Surgery, Samsung Medical Center, Sungkyunkwan University School of Medicine, 81 Irwon-ro, Gangnam-gu, Seoul 06351, Korea

Tel. +82-2-3410-1719, Fax. +82-2-3410-0040

E-mail; yjongman21@gmail.com

https://orcid.org/0000-0002-1903-8354 of HCA to HCC has been reported to be $0.4-6.2 \%{ }^{4-6}$ In previous studies, the risk factors for malignant transformation were tumor size greater than $5 \mathrm{~cm}$, multiple HCA, presence of $\beta$-catenin mutation, and male sex. ${ }^{6,7}$ In Korea, there have been only two cases of HCC arising from HCA in a male patient so far. ${ }^{8,9}$ Therefore, we report a case of HCC within HCA in a normal liver of an elderly male patient. Furthermore, we had difficulties determining treatment because of conflicting imaging and biopsy results.

\section{CASE REPORT}

\section{Clinical findings}

An 80-year-old male patient was transferred to our center for treatment of a 4.5 -cm-sized liver mass in segment 4 that had been incidentally detected on screening ultrasonography and was suspected to be HCC. The patient's medical history included hypertension, diabetes mellitus, chronic kidney dis- 
ease, and acute myocardial infarction that required percutaneous coronary intervention thrice, for which he had been taking clopidogrel for 20 years. The cardiac ejection fraction was $45 \%$ on echocardiography examination. He had no history of alcohol abuse or smoking. His general condition was poor, and Eastern Cooperative Oncology Group performance status was grade 3 , although the physical examination did not reveal any abnormal findings. The laboratory findings were as follows: white blood cell count, 9,030/ $\mu \mathrm{L}$; hemoglobin, $11.0 \mathrm{~g} / \mathrm{dL}$; platelets, $281,000 / \mu \mathrm{L}$; total bilirubin, $0.2 \mathrm{mg} / \mathrm{dL}$; albumin, $4.1 \mathrm{~g} / \mathrm{dL}$; international normalized ratio, 1.06; aspartate aminotransferase, $22 \mathrm{IU} / \mathrm{L}$; alanine aminotransferase, $13 \mathrm{IU} / \mathrm{L}$; blood urea nitrogen, $26.3 \mathrm{mg} / \mathrm{dL}$; and creatinine, $1.78 \mathrm{mg} / \mathrm{dL}$. The alpha-fetoprotein level was $3.3 \mathrm{ng} / \mathrm{mL}$, and protein induced by vitamin $\mathrm{K}$ absence or antagonist-II level was $22 \mathrm{pg} / \mathrm{mL}$. Liver function was preserved (Child-Pugh score, A). Serological tests for hepatitis B surface antigen, antibody to the core antigen, and antibody against hepatitis $\mathrm{C}$ virus were all negative.

\section{Imaging and pathological findings}

Initial contrast-enhanced computed tomography (CT) revealed a fat-containing lesion with no central scar. It also showed heterogeneous arterial enhancement and delayed washout (Fig. 1). Magnetic resonance imaging (MRI) revealed the same findings as $\mathrm{CT}$ with heterogeneous arterial enhancement and delayed washout (Fig. 2). Based on imag-

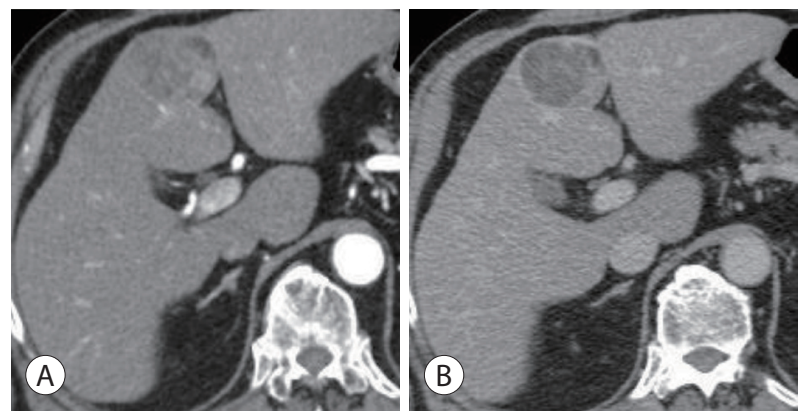

Figure 1. Radiologic findings of the hepatic mass. Initial contrastenhanced computed tomography revealed a $4.5-\mathrm{cm}$-sized mass in segment 4 with heterogeneous arterial enhancement $(A)$ and delayed washout (B). The lesion had no central scar and was compatible with hepatocellular carcinoma. ing, the lesion was suspected to be HCC; however, the decision to operate was difficult because of the patient's age and many risk factors. Ultrasound-guided percutaneous liver biopsy was performed to assist with the treatment plan. The results of the liver biopsy showed that the lesion was consistent with an inflammatory-type liver cell adenoma. Since the results from imaging and biopsy were different, and the risk of surgery was high, we decided to follow up with repeat laboratory and imaging evaluations after 1 year.

\section{Diagnosis and treatment progress (diagnoses of liver disease and liver cancer)}

One year later, the laboratory test results did not change, although imaging tests demonstrated that the size of the mass had increased from $4.5 \mathrm{~cm}$ to $5.5 \mathrm{~cm}$ (Fig. 3). Although the previous biopsy demonstrated HCA, we decided to surgically resect the mass because the findings of the repeat imaging examination were consistent with HCC, and the mass had increased in size.

Since the risk of surgery was high, we chose to perform laparoscopic liver resection rather than open liver resection. Laparoscopic left hemi-hepatectomy was performed for 116 minutes, and the estimated blood loss was $100 \mathrm{~mL}$. The Pringle maneuver was performed once for 15 minutes, and the operation was completed without any remarkable events. The patient was monitored in the intensive care unit on postoperative day 1 and was then transferred to the general ward
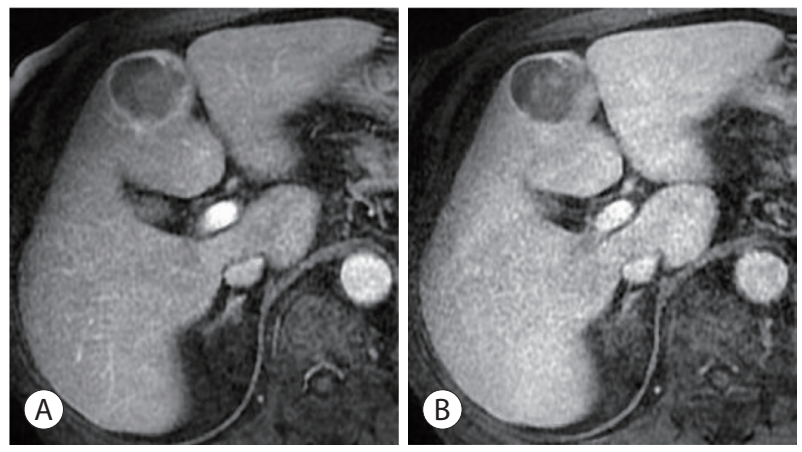

Figure 2. Radiologic findings of the hepatic mass. Magnetic resonance imaging revealed a $4.5 \mathrm{~cm}$-sized mass with heterogeneous arterial reinforcement (A) and delayed loss (B), compatible with computed tomography findings. 
without any remarkable event.

The patient was diagnosed with HCC arising from the HCA. The gross findings of the specimen sections were not typical of HCC (Fig. 4). Microscopy revealed two parts within the serial sections: one part was compatible with adenoma (composed of benign-looking hepatocytes without portal tracts), and the other part was consistent with HCC (increased cellularity) and judged as Edmonson grade 1-2 (Fig. 5). No $\beta$-catenin mutations were detected in the immunohistochemistry assessment.

The patient recovered fully without any complications and was discharged on postoperative day 7 . Follow-up computed tomography performed 3 months after surgery showed no recurrence or complications.

\section{DISCUSSION}

Although its mechanism is poorly understood, the transformation of HCA into HCC is rare. Surgical resection is the preferred treatment for HCA, so there are only a few reports of the transformation of HCA into HCC. ${ }^{4,9,10}$ Compared to female patients taking oral contraceptives, male patients, who have no history of taking hormones, are unlikely to be diagnosed with HCA transformation to HCC. There was one case report of the transformation of HCA into HCC in a male patient without risk factors in Korea, ${ }^{9}$ where the reported risk factors for malignant transformation of hepatic adenoma were tumor size greater than $5 \mathrm{~cm}$, multiple HCA,

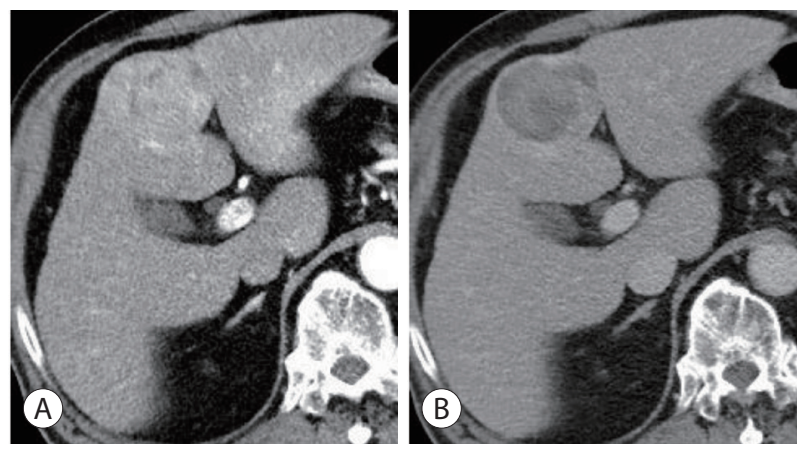

Figure 3. Radiologic findings of the hepatic mass after 1 year of follow-up. The mass increased in size to $5.5 \mathrm{~cm}$, and no other significant changes were noted compared to the previous computed tomography scan (A) arterial phase, (B) delayed phase. presence of $\beta$-catenin mutation, and male sex. ${ }^{6,7}$ In this case, only the male sex was considered as a risk factor at the first visit. However, because the tumor size increased to $>5 \mathrm{~cm}$ at a follow-up examination after 1 year, tumor size and the male sex were included in the list of risk factors.

The imaging studies used for the diagnosis of HCA and HCC include ultrasonography, CT, and MRI. A percutaneous liver biopsy is the gold standard diagnostic tool. However, when imaging studies are conclusive and surgical resection is feasible, percutaneous liver biopsy is not mandatory.

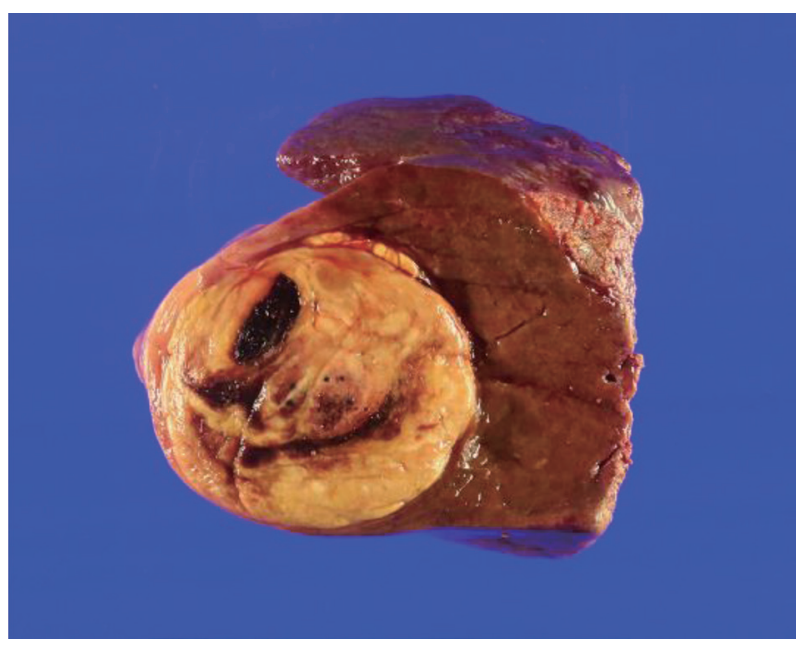

Figure 4. Gross specimen findings of the hepatic mass. The gross findings showed an expanding nodular type and a fat-containing mass.

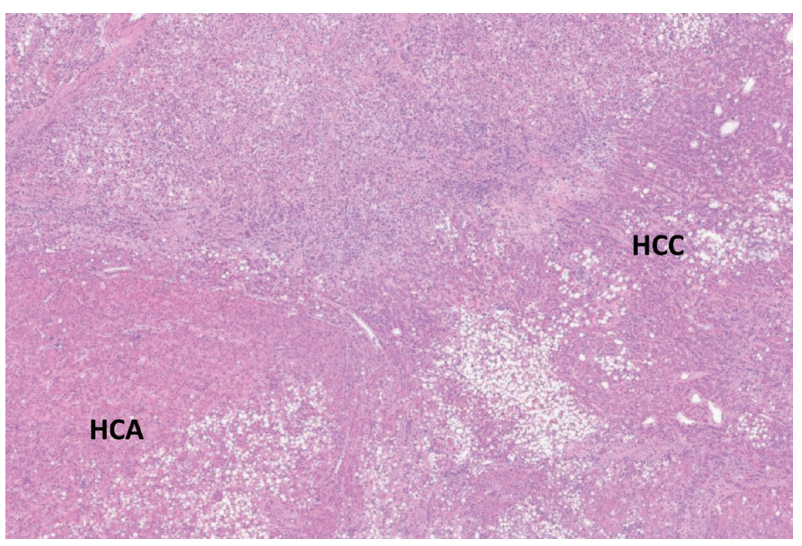

Figure 5. Microscopic pathological findings of the hepatic mass. The hepatocellular adenoma (HCA) part showed an adenoma portion composed of benign-looking hepatocytes without portal tracts, and the hepatocellular carcinoma ( $\mathrm{HCC}$ ) part showed increased cellularity and was judged as Edmonson grade 1-2, consistent with HCC (original magnification, $\times 40$ ). 
In addition, the necessity of biopsy is controversial because it carries a high risk of acute hemorrhage. ${ }^{9,11}$ Nevertheless, immunohistochemical assessment of biopsy specimens considerably enhances the accuracy of diagnosis.

In recent years, genetic modification and identification of signaling pathways in HCA have been developed, and they play an important role in the diagnosis of HCA. ${ }^{12} \beta$-catenin is a subunit of the cell surface cadherin protein complex that acts as an intracellular signal transducer in the WNT signaling pathway. The alteration of $\beta$-catenin activity is associated with the development of HCC and other liver diseases. ${ }^{13}$ Several studies have identified mutations in the $\beta$-catenin gene in HCAs and categorized HCAs into the following four groups according to phenotype and clinical characteristics $\left.{ }^{14-16}: 1\right)$ hepatocyte nuclear factor 1a (HNFla)-mutated (30-50\%), 2) $\beta$-catenin-activated (10-15\%), 3) inflammatory $(35 \%)$, and 4$)$ unclassified tumors $(5-10 \%){ }^{14,17}$ HCC arising from HCA was found in $46 \%$ of $\beta$-catenin-mutated tumors, but it was not observed in inflammatory lesions and, rarely, in HNF1a-mutated tumors. Therefore, $\beta$-catenin activation in HCA is highly associated with its malignant transformation into HCC. ${ }^{12}$

There are two surgical resection methods for liver tumors: open resection and laparoscopic resection. Recently, the use of laparoscopic liver resection has increased due to the development of surgical instruments and techniques. ${ }^{18,19}$ Laparoscopic surgical resection has been reported to be a feasible option with similar efficacy and safety to open resection. In a previous report, laparoscopic resection was associated with significantly less blood loss, less frequent need for pedicle clamping, reduced need for transfusion, and a shorter hospital stay. ${ }^{20}$ Since the patient, in this case, had many risk factors for surgery, laparoscopic liver resection was the better option for tumor removal. Consequently, laparoscopic liver resection was completed in a short time, with less bleeding; the patient was discharged within a short time, as previously mentioned.

In conclusion, although HCA is an uncommon benign tumor of the liver, its transformation into HCC could occur. Therefore, for patients that are male and have a large or increasing mass size, or if $\beta$-catenin is detected, surgical resec- tion is recommended, even if HCA is confirmed by biopsy. If the patient has risk factors for surgery, laparoscopic liver resection should be considered.

\section{ACKNOWLEDGMENTS}

The present case report was approved by the Ethical Committee and Institutional Review Board of Samsung Medical Center (IRB No. 2021-02-154). The operation was performed after obtaining informed consent from the patients.

\section{Conflicts of Interest}

The authors have no conflicts of interest to disclose.

\section{REFERENCES}

1. Baum JK, Bookstein JJ, Holtz F, Klein EW. Possible association between benign hepatomas and oral contraceptives. Lancet 1973;2:926-929.

2. Nault JC, Bioulac-Sage P, Zucman-Rossi J. Hepatocellular benign tumors-from molecular classification to personalized clinical care. Gastroenterology 2013;144:888-902.

3. Belghiti J, Cauchy F, Paradis V, Vilgrain V. Diagnosis and management of solid benign liver lesions. Nat Rev Gastroenterol Hepatol 2014;11:737-749.

4. Yacoub H, Kchir H, Cherif D, Hassine $H$, Haouet S, Ayari A, et al. Hepatocellular carcinoma arising from hepatic adenoma in a young woman. Clin Case Rep 2020;8:1659-1662.

5. Sempoux C, Balabaud C, Bioulac-Sage P. Malignant transformation of hepatocellular adenoma. Hepat Oncol 2014;1:421-431.

6. Farges 0 , Dokmak S. Malignant transformation of liver adenoma: an analysis of the literature. Dig Surg 2010;27:32-38.

7. Dokmak $S$, Paradis V, Vilgrain V, Sauvanet A, Farges $O$, Valla $D$, et al. A single-center surgical experience of 122 patients with single and multiple hepatocellular adenomas. Gastroenterology 2009;137:1698-1705.

8. Choe MS, Yu ES. Hepatocellular carcinoma arising in hepatocelIular adenoma. Taehan Kan Hakhoe Chi 2002;8:107-109.

9. Kim DH, Kim SU, Nam DH, Choi YJ, Park SM, Lee CK, et al. A case of hepatocellular carcinoma within hepatocellular adenoma in a non-cirrhotic male. Korean J Intern Med 2009;24:147-152.

10. Burri E, Steuerwald M, Cathomas G, Mentha G, Majno P, Rubbia-Brandt $L$, et al. Hepatocellular carcinoma in a liver-cell adenoma within a non-cirrhotic liver. Eur I Gastroenterol Hepatol 2006;18:437-441.

11. Grazioli L, Federle MP, Brancatelli G, Ichikawa T, Olivetti L, Blachar 
A. Hepatic adenomas: imaging and pathologic findings. Radiographics 2001;21:877-892.

12. Nault JC, Paradis V, Cherqui D, Vilgrain V, Zucman-Rossi J. Molecular classification of hepatocellular adenoma in clinical practice. J Hepatol 2017;67:1074-1083.

13. Monga SP. Beta-catenin signaling and roles in liver homeostasis, injury, and tumorigenesis. Gastroenterology 2015;148:1294-1310.

14. Zucman-Rossi J, Jeannot E, Nhieu JT, Scoazec JY, Guettier C, Rebouissou S, et al. Genotype-phenotype correlation in hepatocellular adenoma: new classification and relationship with HCC. Hepatology 2006;43:515-524.

15. Dhingra S, Fiel MI. Update on the new classification of hepatic adenomas: clinical, molecular, and pathologic characteristics. Arch Pathol Lab Med 2014;138:1090-1097.

16. Bioulac-Sage P, Rebouissou S, Thomas C, Blanc JF, Saric J, Sa Cunha $A$, et al. Hepatocellular adenoma subtype classification using molecular markers and immunohistochemistry. Hepatology
2007:46:740-748.

17. Stoot JH, Coelen RJ, De Jong MC, Dejong CH. Malignant transformation of hepatocellular adenomas into hepatocellular carcinomas: a systematic review including more than 1600 adenoma cases. HPB (Oxford) 2010;12:509-522.

18. Kim Y, Amini N, He J, Margonis GA, Weiss $\mathrm{M}$, Wolfgang $\mathrm{CL}$, et al. National trends in the use of surgery for benign hepatic tumors in the United States. Surgery 2015;157:1055-1064.

19. Cho W, Kwon CHD, Choi JY, Lee SH, Kim JM, Choi GS, et al. Impact of technical innovation on surgical outcome of laparoscopic major liver resection: 10 years' experience at a large-volume center. Ann Surg Treat Res 2019;96:14-18.

20. Landi F, De' Angelis N, Scatton O, Vidal X, Ayav A, Muscari F, et al. Short-term outcomes of laparoscopic vs. open liver resection for hepatocellular adenoma: a multicenter propensity score adjustment analysis by the AFC-HCA-2013 study group. Surg EndosC 2017;31:4136-4144. 